\title{
Accurate Assessment of Link Loss Rate in Wireless Mesh Networks
}

\author{
Bing Qi, Saad Biaz \\ qibing1@auburn.edu, biazsaa@auburn.edu \\ Technical Report CSSE08-02 \\ Dept. of Computer Science and Software Engineering \\ Auburn University, AL, 36849-5347, USA
}

August 19, 2008

\begin{abstract}
A number of routing metrics have been proposed to select a path with better performance in wireless mesh networks. Most of them partially rely on link loss rates when evaluating the path's performance. Thus accurately measuring the link loss rate is essential to the design of effective routing metrics identifying efficient paths. Currently, a broadcast based probe mechanism is commonly used by routing metrics to estimate the link loss rates. However, this mechanism often yields inaccurate link loss rates. Based on our extensive simulations, we find out that the limitations of this mechanism are due to: 1) the use of basic (lowest) data rate to probe the loss rate while the link might be used with a higher data rate; 2 ) the assumption that links are symmetric, which is not true in real case; 3) the low number of packet probes used to measure the link loss rate. This work proposes appropriate solutions to the above weaknesses. Our experimental results indicate that our proposed modifications can measure the link loss rate accurately.
\end{abstract}

\section{Introduction}

A Wireless Mesh Network (WMN) is a communication network that consists of independent wireless nodes [1]. It is dynamically self-organized and self-configured, with the nodes in the network automatically establishing and maintaining node connectivity among themselves. Unlike traditional mobile ad-hoc networks, the mesh nodes have minimal mobility and the topology normally does not change. Therefore, a WMN can be seen as a special type of wireless ad hoc network. 
Due to these unique features, WMN has gained considerable popularity recently both in research and industry. A number of interesting applications or systems have emerged and some are commercially available based on such wireless networks [2-4]. Many on-going research projects aim to enhance WMN's performance. A key focus of this research is to determine high-throughput paths. Traditional wired networks have stable communication links and identical bandwidths, thus locating a path with the minimum hop count metric may suffice to guarantee the high-throughput performance. However, unlike wired networks, WMNs have wireless links with a wide range of different link loss rates or data transmission rates. Therefore, the design of an effective routing metric is more complex and challenging. To design such a metric, it is necessary to capture all the characteristics of the wireless links and paths that might affect the path performance. The more path characteristics the metric catches, the higher performance it will be. Among all these link characteristics, link loss rate is one of the key properties to affect link and path performance.

Many link quality aware routing metrics have been proposed such as the Expected Transmission Count (ETX) [5], Round Trip Time (RTT) [6], the Expected Transmission Time (ETT) [7], and Expected Transmission Time (iETT) [8]. Most of them consider the link loss rate. For example, the Expected Transmission Count (ETX) evaluates paths largely based on the link loss rate, and Expected Transmission Time (ETT) actually is the function of the loss rate and the bandwidth on each link, which represents the total transmission time needed to send a data packet successfully over a path. Therefore, it is critical to assess the link loss rate accurately in order to guarantee the effectiveness of these routing metrics.

Currently, most routing metrics use a broadcast based probe method proposed by De Couto et al. to measure the link loss rate [5]. In this method, each node broadcasts a link probe packet every second, and every neighbor tracks the number of probes received in the last ten seconds to approximately measure the link loss rate. However, this method often yields inaccurate measurement of link loss rates.

In this report, we analyze and evaluate the traditional method of measuring the link loss rate, and point out its weaknesses and drawbacks: 1) the probes are sent out with the basic (lowest) data rate which is not the rate used for sending data packets; 2) this method assumes that links are symmetric, i.e., that loss rates are the same in both directions; 3) the frequency of sending out probe packets is one per second over 10 seconds generates a too small number of probes to get accurate link loss rates.

The rest of this paper is organized as follows: Section 2 summarizes the motivation of our work and describes the traditional method of measuring link loss rate in detail. In Section 3, we examine the shortcomings of this method and propose corresponding solutions. Section 4 demonstrates the experimental settings and results. The conclusions are presented in Section5. 


\section{Motivation and Related Work}

In Wireless Mesh Networks (WMNs), nodes communicate with wireless signals. Due to the shared nature of these communication channels, the packet loss are ineluctable. Based on 802.11 protocol [9], the MAC layer uses link-level retransmissions for frame losses. Thus it takes more time to successfully send a packet over a lossy link. This retransmission time not only negatively affects this specific link performance, it also hurts the overall path throughput because other links on the route cannot send any packet while the sender is retransmitting. As a result, these link losses will be felt at application layer in forms of lower throughput and higher end-to-end delays. Therefore, it is essential to accurately capture the loss rates of the links, and avoid highly lossy links when selecting a routing path.

In order to accommodate different channel conditions, it is possible to use different data transmission rates based on different modulation schemes. This multi-rate characteristic has been shown to greatly improve wireless multi-hop network performance [10-12]. So, modern wireless radios typically support different rates. Therefore, a wide range of data transmission rates exists simultaneously within one wireless mesh network. Due to the physical properties of wireless radios, a higher data rate usually requires a better channel in order to transmit packets successfully. So on average, sending a packet with a lower data rate will more likely be delivered. Most existing routing protocols used in wireless networks, such as DSR [13], AODV [14], and LQSR [7], discover routing paths with a broadcast based method. These routing protocols broadcast route request packets into networks using the basic (lowest available) data transmission rate. As a result, the routing packets sent from the source node normally can be received successfully in the destination node. However, in a wireless mesh network which supports multi-rate, those paths acquired by routing protocols might not be efficient enough to support the actual data transmissions (in general higher than the basic data rate), because the actual higher data rate will yield higher loss rates

resulting in higher retransmission times. Thus it is necessary to consider an accurate link loss rate when determining a routing path.

A simple method used by most current routing metrics to measure individual link loss rate was proposed by De Couto et al [5]. We refer this method as Broadcast-Based Probing method (BBP) in this study. According to BBP, each node periodically broadcasts a link probe packet to its neighbor nodes at an average period of $\tau$. In order to avoid synchronization, probes are sent out with a jitter of $0.01 * \tau$. Wireless nodes are required to record the number of probes they have received during the last $\omega$ seconds. Therefore the delivery rate from the sender at time $t$ can be represented as the follows:

$$
d(t)=\frac{\operatorname{count}(t-\omega, t)}{\omega / \tau}
$$


Here count $(t-\omega, t)$ is the number of probes received during the window $\omega$, and $\omega / \tau$ is the number of probes that should have been received.

Since most wireless links are bidirectional, probes' loss rates on both directions are considered to measure the loss rates in this BBP method. Therefore, in addition to tracking the number of probe packets received, every node needs to put its measured loss rates in its own probe packets and sends them to its neighbors. Since the broadcast packets are neither re-transmitted nor acknowledged according to IEEE802.11 technologies, a node can measure link loss rates based on the information obtained from its neighbors and it own records. Given one link A to B, let suppose $d_{f}$ be the forward probe packets' delivery ratio $\left.(A \rightarrow B)\right)$ and $d_{r}$ be the probability of the successfully reverse delivery rate $(B \rightarrow A)$. Then the probability that a data packet can be successfully transmitted via link $A B$ is:

$$
p=\frac{1}{d_{f} * d_{r}}
$$

Consequently, the link loss rate for this specific link is $1-p$.

\section{Limitations and our Solutions}

Although the BBP method is widely used by current routing metrics in wireless mesh networks, it has some limitations that may yield inaccurate loss rates. In this section, we demonstrate BBP drawbacks using the $n s-2$ simulator [15] and explain the reasons for these drawbacks.

The first drawback of the BBP is that it assumes the loss rate of packets sent by the basic data rate is the same as the loss rate for packets sent with other higher data transmission rates: BBP broadcasts probe packets with the basic data rate and measures the frame loss rate for that basic rate. This assumption is not valid. There is some relationship between the bit error rate (directly relevant to the loss rate of link) and signal-to-noise (SNR) for various modulation schemes or data rates [10]. For a given SNR, the modulation scheme with a higher data rate usually yields a higher loss rate, as shown in Figure 1 [10]. In other words, given a specific link, sending packets with lower data rates is more reliable (less link loss) than sending packets with a higher data transmission rate.

Wireless Mesh Networks normally use radios supporting multiple rates. Therefore, depending on channel conditions, distance and modulation schemes, different links will have very different transmission rates. Currently, the IEEE 802.11g standard offers data rates of 6, 9, 12, 18, 24, 36, 48 and 54 Mbps while the earlier IEEE 802.11b standard supports 1, 2, 5.5 and 11 Mbps data rates $[16,17]$. The difference of data rates is more prominent in future IEEE 80211n standard, in which data rates will be in the ranging of $6 \mathrm{Mps}$ to $200 \mathrm{Mbps}$. According to the parameters from the published specifications of many wireless card providers, such as Lucent ORiNOCO PC 


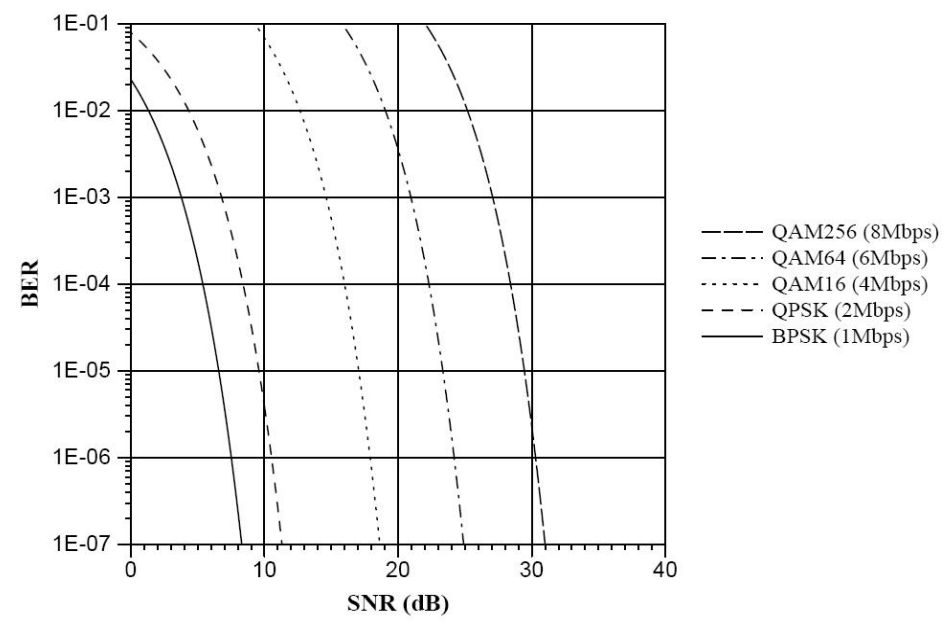

Figure 1: BER and as a function of SNR

card [18], a commonly used IEEE $802.11 \mathrm{~b}$ wireless card, the sensitivities for successfully received data packets vary for different transmission rates. Therefore, using the basic data rate to probe the link loss rates is not effective on multi-rate networks. As a result, BBP tends to underestimate the loss rates for wireless links. To address this drawback, we propose to send probes with the actual data transmission rates when we probe the link loss rates for wireless links.

The second limitation is that BBP computes the link loss rate based on its probes' loss rates in both directions of that link. According to the equation 2 in previous section, BBP assumes that the link is symmetric. Thus for a bidirectional link $A B$, the link $A \rightarrow B$ and $B \rightarrow A$ hold exactly the same link loss rate with this method. Nonetheless, in real wireless network scenarios, a substantial percentage of wireless links are asymmetrical especially when the distance between nodes is large [19]. So BBP method omits the asymmetric nature of wireless links.

Furthermore, the successful delivery of a packet requires successful forward delivery and successful acknowledgement delivery. The link loss rate depends on both directions. However, based on IEEE 802.11 standards [9], a receiver sends the ACK at the basic data rate. So ACKs have higher probabilities to be transmitted successfully than normal data packets because data packets are sent with the real data transmission rate. If a data packet can be transmitted successfully in forward link with higher data rates, the chance for the ACK to get through (on the reverse direction) is very high. To take wireless links' asymmetry and ACKs transmitted with basic data transmission rate issues into account, it is more appropriate to count only one way link probes to measure the link loss rates. By doing this, the link loss rate from A to B is different from the link loss rate from B to A.

The third limitation of BBP lies in its implementation. BBP sends probes every second (interval) to its neighbors, and the time (duration/window size) of sending probes is 10 seconds. To 


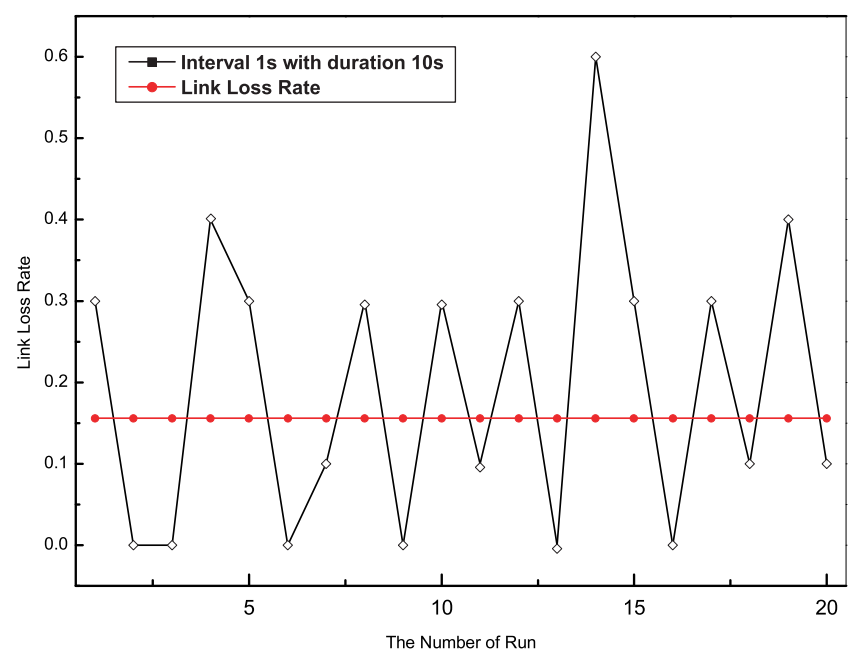

Figure 2: Measured Link Loss Rate with Interval 1 Second

verify whether these parameters (interval and duration) are reasonable enough to measure the link loss rate accurately, the following experimental settings are established. Two wireless nodes (S and D) are placed at a distance of 250 meters. Both Node S and Node D are configured with one wireless radio supporting $802.11 \mathrm{~g}$. To find out whether interval one second with 10 seconds duration is appropriate to get accurate loss rates, we conduct experiments with interval 1 to probe the link loss rate from Node $\mathrm{S}$ to Node D. In this experiment, the actual data transmission rate is used to send probes and the probing duration is set to 10 seconds. 20 separate experiments are conducted. For each experiment, one individual link loss rate value is obtained. Figure 2 plots the link loss rates achieved from these 20 independent experiments, each point represents one experimental result. In this figure, the vertical axis represents the link loss rates and the horizontal axis stands for the experiment number. The line marked with $\bullet$ indicates the real loss rate of the link, the line marked with $\diamond$ is the loss rates measured by these 20 independent experiments. According to the results, $\mathrm{BBP}$ is prone to lead to a wide range of different loss rates from 0.0 to 0.6 for measuring one specific link. Apparently, the loss rates obtained by a one second interval with a duration of 10 seconds are inaccurate. Given the same link, the measured loss rate is not consistent and in real case only one measurement is used.

To detect how different intervals affect the accuracy of link loss rate measurements, various interval parameters are tested, which include $1 \mathrm{~s}, 0.5 \mathrm{~s}, 0.25 \mathrm{~s}, 0.1 \mathrm{~s}, 0.05 \mathrm{~s} 0.025 \mathrm{~s}, 0.01 \mathrm{~s}$, and $0.005 \mathrm{~s}$. Like the former experiments, the actual data transmission rate is used to send probe packets and the duration is set to 10 seconds. According to our results, the interval $0.5 \mathrm{~s}$ and $0.1 \mathrm{~s}$ are still not effective enough to measure link loss rate accurately. Interval $0.005 \mathrm{~s}$ is effective. To make our the figure clearer, Figure 3 only plots the correspondent link loss rates acquired by intervals $0.25 \mathrm{~s}$, $0.05 \mathrm{~s}, 0.025 \mathrm{~s}$ and $0.01 \mathrm{~s}$. Observe the experimental results from this figure, link loss rates can be calculated precisely when setting intervals to $0.025 \mathrm{~s}$ or $0.01 \mathrm{~s}$. Although setting interval 0.01 is 


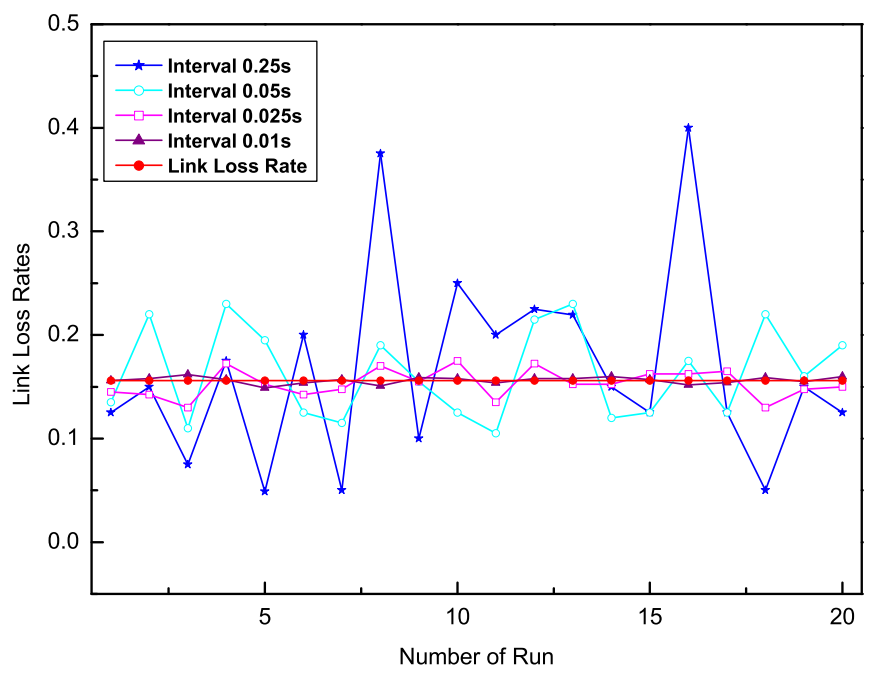

Figure 3: Measured Link Loss Rate with Different Interval

more precise than using interval 0.025 , the result with $0.025 \mathrm{~s}$ is already reliable enough since the maximal deviation getting from interval $0.025 \mathrm{~s}$ is only 0.015 .

Another way to measure the link loss rate more accurately is to use durations(windows) longer than 10 seconds. Actually, the accuracy of the link loss rate significantly depends on the number of probes sent out. If only a total of 10 probes is sent out (interval of $1 \mathrm{~s}$ and the duration is $10 \mathrm{~s}$ ), whether one probe packet is lost greatly affects the final link loss rate. However if we send more probes in one unit time or in a longer duration, one or two single packet's loss will have a small effect on the overall result, which roughly leads to more accurate measurements. For example, Figure 4 demonstrates the link loss rate measured by using an interval of 1 s with a duration of 400 second to test the link loss rates with 20 separate experiments. Obviously, longer duration yields better accuracy.

\section{Experimental Setting and Results}

In this section, the effectiveness of our proposed solutions for BBP's drawbacks is evaluated using $n s$-2 simulator [15]. To make our experimental setting close to real systems, the parameters used in our simulator are based on the specification published on Cisco website [20]. Table 4 lists the sensitivities used for each individual transmission rate. Two wireless nodes (source Node $\mathrm{S}$ and destination Node D) are used in our simulation; the distance between the two nodes is set to vary from 210 to 300 meters in steps of 10 meters (according to the parameter shown in table 1, data rate transmitted with this range is typically $24 \mathrm{Mbps}$ ). Within each wireless node, an IEEE $802.11 \mathrm{~g}$ 


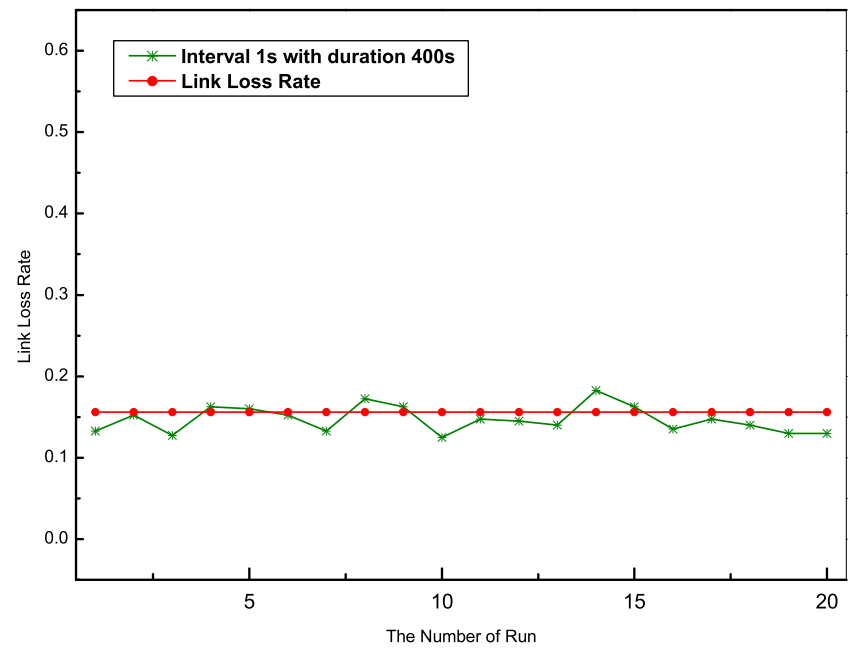

Figure 4: Measured Link Loss Rate with Longer Duration

radio is configured.

For each distance between Node $\mathrm{S}$ and Node D, we use the following five protocols to measure the link loss rates independently. 1) Original BBP: it uses the basic data rate to send probes with an interval of 1 second in a duration of 10 seconds, and the link loss rate is measured based on probe delivery rates in both directions; 2) BBP-I: it uses the basic data rate to send probes with an interval of 0.025 second in a duration of 10 seconds, and the link loss rate is measured based on both directions; 3) BBP-II: it uses the actual data rate to send probes with an interval of 1 second in a duration of 10 seconds and the link loss rate is measured based on the number of received packets in only the forward direction; 4) BBP-III: it uses the actual data rate to send probes with an interval of 0.025 second in a duration of 10 seconds and the final link loss rate is measured based on both directions of that link; 5) BBP-final, it is a BBP protocol with all modifications. BBP-final adopts all the proposed solutions explained in the previous section. So the difference between BBP-final and BBP-III is that BBP-final method measures the link loss rate only based on the number of received probes in forward direction. The actual link loss rate is measured by a real CBR traffic initiated from Source node $S$ to Destination node D. Since 802.11 MAC scheme uses link level acknowledgement and retransmission mechanisms to mask data packet losses for the application layer. So from the application layer point of view, links are packet-loss free with the price of low throughput. To calculate the actual link loss in real applications, we disabled this retransmission scheme. By checking the trace file, the actual link loss rate can be successfully and precisely measured.

The experimental results are shown in Figure 5. Here the vertical axis represents the link loss rates measured and the horizontal axis stands for the distances between these two nodes. Totally 


\begin{tabular}{|c|c|}
\hline Parameter & Description \\
\hline Transmission Power & $10 \mathrm{dbm}$ \\
\hline 54Mbps Sensitivity & $-68 \mathrm{dbm}$ \\
\hline 48Mbps Sensitivity & $-71 \mathrm{dbm}$ \\
\hline 36Mbps Sensitivity & $-75 \mathrm{dbm}$ \\
\hline 24Mbps Sensitivity & $-79 \mathrm{dbm}$ \\
\hline 18Mbps Sensitivity & $-82 \mathrm{dbm}$ \\
\hline 12Mbps Sensitivity & $-84 \mathrm{dbm}$ \\
\hline 9Mbps Sensitivity & $-87 \mathrm{dbm}$ \\
\hline 6Mbps Sensitivity & $-89 \mathrm{dbm}$ \\
\hline
\end{tabular}

Table 1: Wireless Card Interface Parameter

six curves are plotted in this figure, and the red curve marked with $\bullet$ is the real link loss rates for each specific distance. The result measured with the original BBP sending probes with basic data rate is shown by the curve marked with $\star$ that obviously underestimates the link loss rates, because the required sensitivities to receive a packet with the basic data rate is much lower than the sensitivities needed to decode a packet sent with a higher data transmission rate. The same conclusion can be drawn from the curve marked with $\triangle$, which also sends probes with the basic data rate. Meanwhile, as revealed by the curve marked with $\circ$, when sending probes with actual data transmission rates, the number of probes is also an important factor to affect the accuracy of the link loss rates' assessment. The BBP implementation uses one second as interval and 10 seconds as duration. These values are not effective to get accurate link loss rates. We also find that calculating the loss rate based on the link quality of both directions leads to overestimating the real link loss rate according to the curve marked with $\diamond$. As a conclusion, to address all the drawbacks, our solution measures the link loss rate accurately, as shown by the curve marked with $*$.

\section{Conclusions}

In this study, Broadcast Based Probe method (BBP), a commonly used method adopted by many routing metrics to measure the link loss rates, has been extensively investigated. Based on $n s-2$ simulations, we found that the accuracy of BBP is low in wireless networks supporting multiple rates. The limitations of the BBP method are mainly due to overall three aspects: 1) the probes are sent out with the basic data rate; 2) Deriving the link loss rate based on the link quality of both directions of the link is not necessary; 3 ) The number of probes(one second interval with window size 10 second) is not sufficient to deliver accurate loss rates.

To take all the limitations into consideration, we propose 1) to use actual data rates when sending probe packets to neighbors, 2) to send more probes, and 3) to compute the loss rate based 


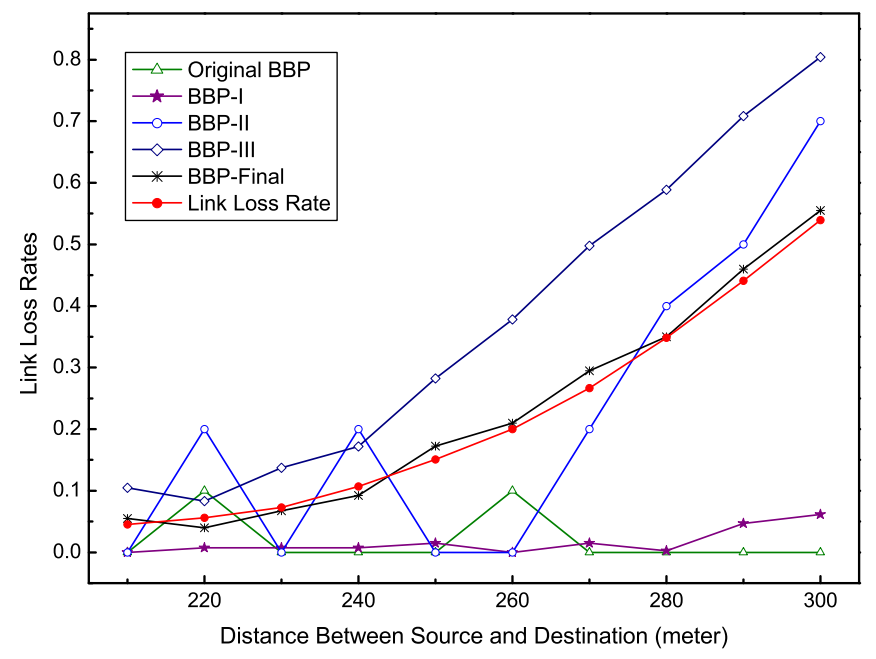

Figure 5: Link Loss Rates Based on Different Measure Protocols

on the forward link only. Our simulation results suggest that these modifications make BBP more accurate.

\section{References}

[1] I. F. Akyildiz, X. Wang, and W. Wang, "Wireless mesh networks: a survey," Computer Networks, vol. 47, no. 4, pp. 445-487, March 2005. [Online]. Available: http://portal.acm.org/citation.cfm?id=1071646

[2] R. Karrer, A. Sabharwal, and E. Knightly, "Enabling large-scale wireless broadband: The case for taps,” 2003. [Online]. Available: citeseer.ist.psu.edu/karrer03enabling.html

[3] “Bay area wireless user group.” [Online]. Available: http://www.bawug.org/

[4] "Mit roofnet." [Online]. Available: http://www.pdos.lcs.mit.edu/roofnet

[5] D. DeCouto, D. Aguayo, J. Bicket, and R. Morris, "A high-throughput path metric for multihop wireless routing," in MobiCom '03: Proceedings of the 9th annual international conference on Mobile computing and networking. New York, NY, USA: ACM Press, 2003, pp. 134-146.

[6] A. Adya, P. Bahl, J. Padhye, A. Wolman, and L. Zhou, "A multi-radio unification protocol for ieee 802.11 wireless networks," in BROADNETS '04: Proceedings of the First International 
Conference on Broadband Networks (BROADNETS'04). Washington, DC, USA: IEEE Computer Society, 2004, pp. 344-354.

[7] R. Draves, J. Padhye, and B. Zill, "Routing in multi-radio, multi-hop wireless mesh networks,' in MobiCom '04: Proceedings of the 10th annual international conference on Mobile computing and networking. New York, NY, USA: ACM Press, 2004, pp. 114-128.

[8] S. Biaz, B. Qi, and Y. Ji, "Improving expected transmission time metric in multi-rate multihop networks," Consumer Communications and Networking Conference, 2008. CCNC 2008. 5th IEEE, pp. 533-537, Jan. 2008.

[9] "IEEE 802.11 Local and Metropolitan Area Networks: Wireless LAN Medium Acess Control (MAC) and Physical (PHY) Specifications.” 1999. [Online]. Available: http://grouper.ieee.org/groups/802/11/main.html

[10] G. Holland, N. H. Vaidya, and P. Bahl, "A rate-adaptive MAC protocol for multi-hop wireless networks," in Mobile Computing and Networking, 2001, pp. 236-251. [Online]. Available: citeseer.ist.psu.edu/holland01rateadaptive.html

[11] B. Sadeghi, V. Kanodia, A. Sabharwal, and E. Knightly, "Opportunistic media access for multirate ad hoc networks," 2002. [Online]. Available: citeseer.ist.psu.edu/sadeghi02opportunistic.html

[12] B. Awerbuch, D. Holmer, and H. Rubens, "Effects of multi-rate in ad hoc wireless networks." [Online]. Available: citeseer.ist.psu.edu/676530.html

[13] D. B. Johnson and D. A. Maltz, "Dynamic source routing in ad hoc wireless networks," in Mobile Computing, Imielinski and Korth, Eds. Kluwer Academic Publishers, 1996, vol. 353. [Online]. Available: citeseer.ist.psu.edu/johnson96dynamic.html

[14] C. E. Perkins and E. M. Royer, “Ad-hoc on-demand distance vector routing," wmcsa, vol. 00, p. $90,1999$.

[15] “The ns-2 simulator.” [Online]. Available: http://www.isi.edu/nsnam/ns/

[16] "Supplement to IEEE standard for information technology telecommunications and information exchange between systems - local and metropolitan area networks - specific requirements. part 11: wireless LAN Medium Access Control (MAC) and Physical layer (PHY) specifications. Amendment 2: higher-speed physical layer (PHY) extension in the $2.4 \mathrm{GHz}$ band." 1999. 
[17] "IEEE Std 802.11h-2003 (Amendment to IEEE Std 802.11, 1999 edn. (Reaff 2003)) as amended by IEEE Stds 802.11a-1999, 802.11b-1999, 802.11b-1999/cor 1-2001, and 802.11d-2001)." 2003.

[18] "The hardware specification of orinoco card." [Online]. Available: http://www.agere.com/client/wlan.html

[19] D. Kotz, C. Newport, and C. Elliott, "The mistaken axioms of wireless-network research," Dartmouth College, Tech. Rep., July 2003.

[20] "Cisco aironet $802.11 \mathrm{a} / \mathrm{b} / \mathrm{g}$ wireless cardbus adapter." [Online]. Available: http://www.cisco.com/en/US/prod/collateral/wireless/ps6442/ps4555/ps5818/ 